\title{
The effect of low energy electron and UV/VIS radiation aging on the electron emission properties and breakdown of thin-film dielectrics
}

\author{
J. R. Dennison*, C. D. Thomson, and A. M. Sim \\ Utah State University, Physics Department \\ 4415 Old Main Hill, Logan, Utah, 84322-4415, USA \\ * E-mail: JR.Dennison@usu.edu
}

\begin{abstract}
Studies of secondary and backscattered electron yield curves of thin-film dielectrics have recently been made using pulsed, low current electron beam methods to minimize insulator charging. These capabilities have allowed us to investigate the evolution of surface and internal charge profiles as a function of low energy electron ( $<1 \mathrm{keV}$ to $20 \mathrm{keV}$ ) pulsed-electron fluence to determine how quickly insulators charge, and how this can affect subsequent electron emission properties. We have also studied critical incident electron energies that result in electrical breakdown of insulator materials and the effect of breakdown on subsequent emission, charging and conduction. The qualitative physics of such processes in solid dielectrics has long been known; this work begins to place such studies on a quantitative basis.
\end{abstract}

\section{INTRODUCTION}

The yields and energy spectra of electrons emitted due to electron, ion and photon bombardment are key to modeling the extent of spacecraft charging (SCC) [1]. The emitted electrons can be divided into two categories: (i) secondary electrons (SE) $(<50 \mathrm{eV}$ by convention) that originate within the material, and (ii) backscattered electrons (BSE) ( $>50 \mathrm{eV}$ by convention) that originate from the incident electron source $[2,3]$. SE and BSE yields are defined as the ratios of the emitted electrons to the total incident electrons.

Of particular importance for SCC are emission characteristics of insulators [4,5]. However, accurate absolute electron yield measurements on dielectrics are much more difficult to make than on conductors, since any charge that is deposited in the material is not highly mobile and cannot easily be neutralized. The surface and bulk potentials and internal charge distributions that develop as a result of electron bombardment can subsequently affect electron emissions by influencing incident electron energies, or by creating electric fields that affect the escaping SE's and BSE's. Without the implementation of neutralization techniques, an irradiated insulator will eventually charge to a (quasi-) steady state current equilibrium such that the net current to the sample approaches 0 or the total electron yield is $\sim 1[2,3]$.

The DC and pulsed electron yield methods used here are described fully elsewhere [5-7]. For pulsed measurements on insulators, the electron guns delivered $5 \mu \mathrm{s}, 20-60 \mathrm{nA}$ incident pulses with $\sim 10^{6}$ electrons/pulse. A low-energy $(<1 \mathrm{eV})$ electron flood gun was used to neutralize positive surface charging between pulses [6-8]. Numerous conductor and insulator electron spectra, yield curves, and yield parameters have been measured at Utah State [4,7,9].

Measurements on two common spacecraft insulators are reported here; additional measurements on these samples are found elsewhere [4-6]. Measurements of a chromic acid anodized $\mathrm{Al}$ alloy (Al2219) sample (2 mm thick, $10 \mathrm{~mm}$ diameter with a $1.3 \mu \mathrm{m}$ chromic acid anodized surface coating on each side) are shown in Fig. 1. This material is used throughout the International Space Station body as a structural material and for micrometeriod and orbital debris shielding [10]. The Kapton $^{\mathrm{TM}}$-aluminum sample (see Fig. 2) was a composite material sold by Sheldahl Technical Materials for applications as a low emissivity thermal control coating material for spacecraft. The $8 \mu \mathrm{m}$ thick $10 \mathrm{~mm}$ diameter polyimide Kapton $\mathrm{H}^{\mathrm{TM}}$ substrate was vapor coated with a $\sim 0.1 \mu \mathrm{m} \mathrm{Al}$ backing. Both samples were cleaned using acetone and methanol before introduction into a vacuum chamber operating at $10^{-7} \mathrm{~Pa}$, but were not ion sputtered.

\section{INTERNAL CHARGE DISTRIBUTIONS}

This paper focuses on the effects of charging and internal charge buildup on electron emission. The polarity of insulator charging is dependent on incident electron energies. Positive (negative) charge will build up when the total number of electrons leaving the insulator sample is greater than (less than) the total number of incoming electrons. If the incident electron energy is below $E_{1}$, ( $<100 \mathrm{~V}$ for most good insulators) negative charging results, since few SE's are excited by absorbed electrons. Fig. 1(e) shows a schematic of the internal charge distribution for an energy above $E_{2}(>1$ $\mathrm{keV}$ for most insulators), with a large, deeply embedded negative charge as a result of the large penetration depth of the higher energy incident electrons (up to several microns), exciting SE's (escape length tens of nanometers) that are too deep to escape from the material. As the negative charge builds up, the resulting electric field reduces the energy of additional 


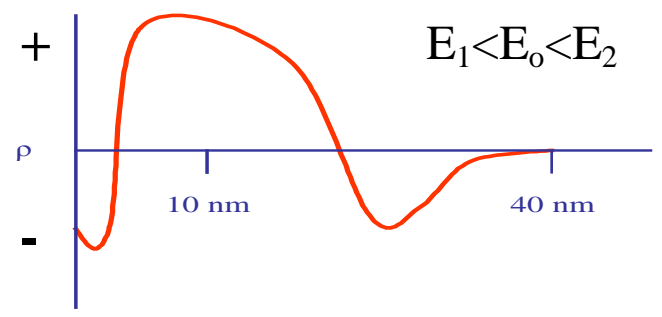

(a)

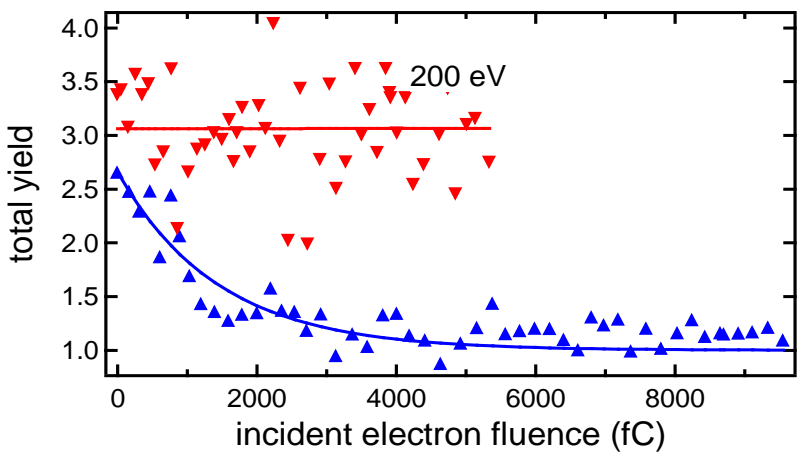

(b)

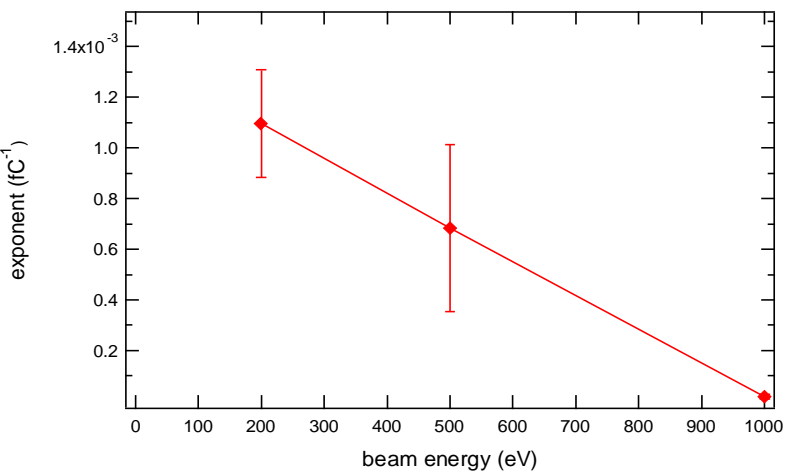

(c)

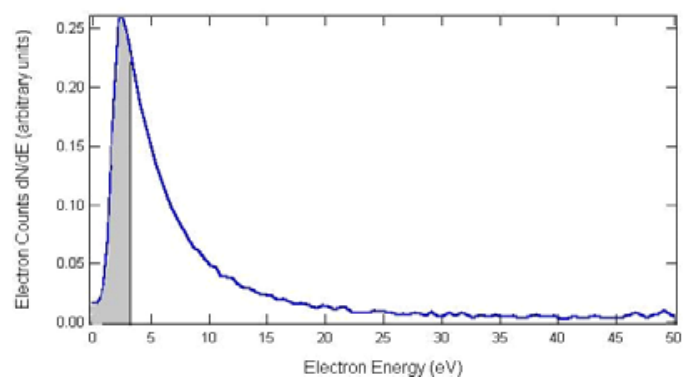

(d)

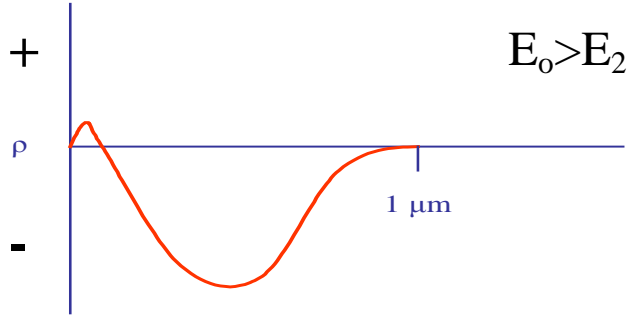

(e)

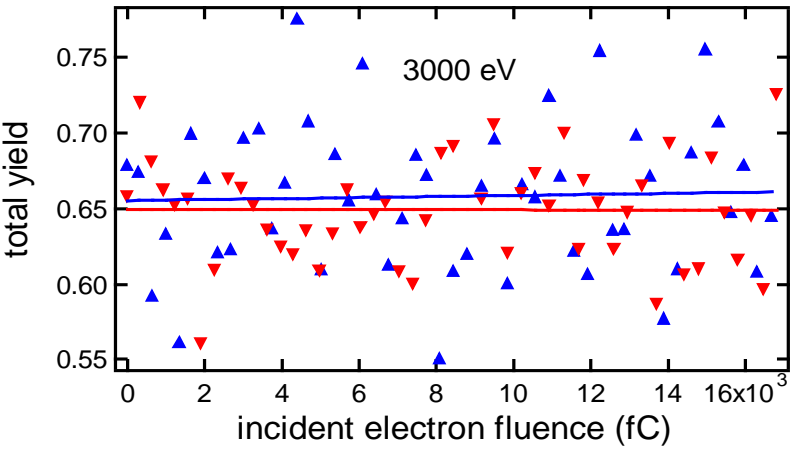

(f)

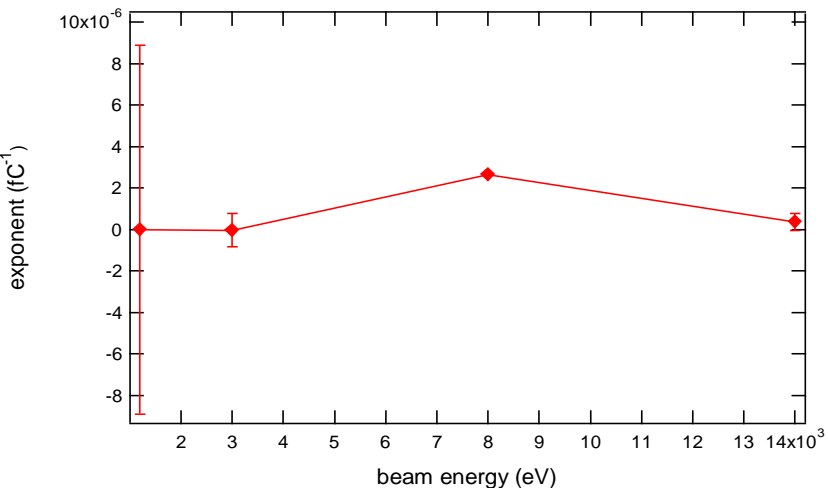

(g)

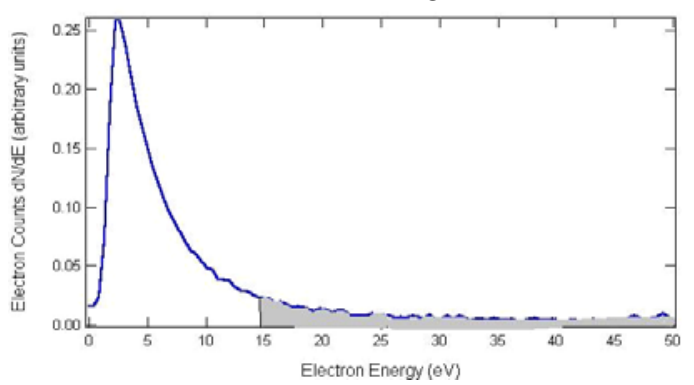

(h)

Fig. 1. Effects of internal charge distributions for (a-d) $E_{1}<E_{0}<E_{2}$ with $\sigma>1$ and overall positive charging and (e-h) $E_{0}>E_{2}$ with $\sigma<1$ and overall negative charging. For the Kapton ${ }^{\mathrm{TM}}$ sample, the total yield crossover energies $\mathrm{E}_{1} \approx 31 \mathrm{eV}$ and $\mathrm{E}_{2} \approx 965 \mathrm{eV}$ [5]. (a) and (e) Schematics of internal charge distributions. (b) and (f) Evolution of total yield as a function of pulsed incident electron fluence at (b) $200 \mathrm{eV}$ between $\mathrm{E}_{1}$ and $\mathrm{E}_{2}$ and (f) $3000 \mathrm{eV}$ above $\mathrm{E}_{2}$. Successive symbols are for consecutive pulses with $(\boldsymbol{\nabla})$ and without $(\boldsymbol{\Delta})$ charge neutralization with low energy electron flooding between pulses. Fits are for exponential decay of the total yields to (quasi-)steady state values [5]. Similar curves at additional energies are found in [5]. (c) and (g) show the total yield decay constant as a function of beam energy. The shaded regions of a typical SE emission spectrum show emitted SE affected by positive (d) and negative (h) charge build up.

incident electrons and inhibits their range. In addition, there is a depletion region of small positive net charge near the surface where low energy SE's can escape the surface assisted by the electric field from the large negative charge distribution.
For incident electrons with $\mathrm{E}_{1}<\mathrm{E}_{0}<\mathrm{E}_{2}$, more electrons will be emitted from the insulator than are incident, and net positive charging will occur. Incident electron penetration is only somewhat larger than the SE escape depth, resulting in a small deep negative charge region and a larger positive charge region closer to the surface 


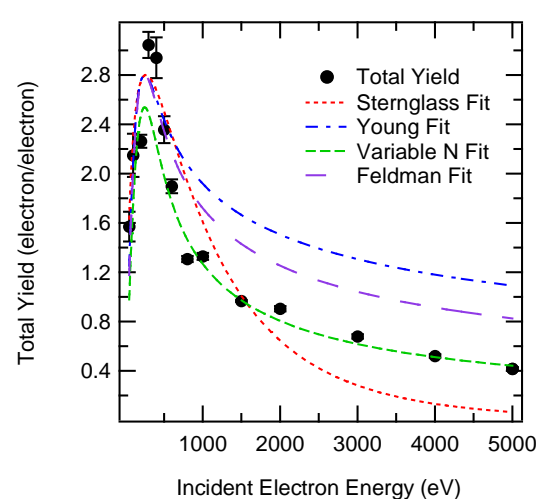

(a)

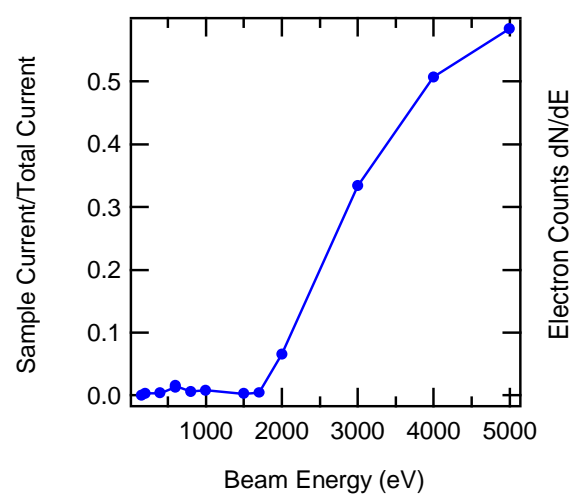

(d)

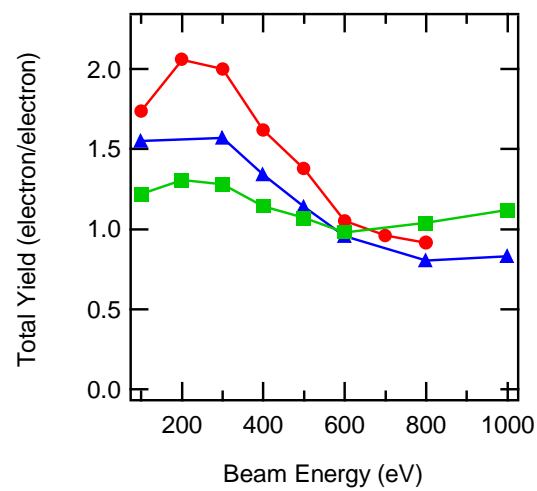

(b)

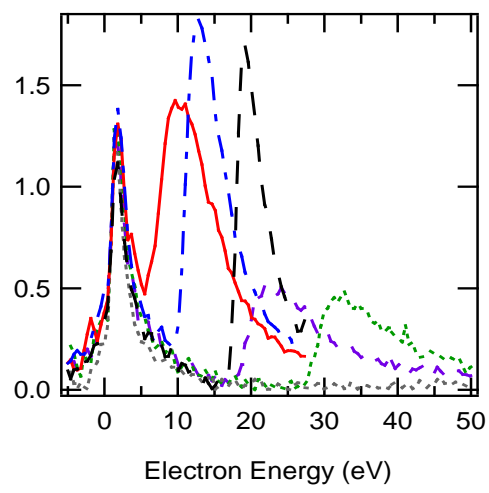

(e)

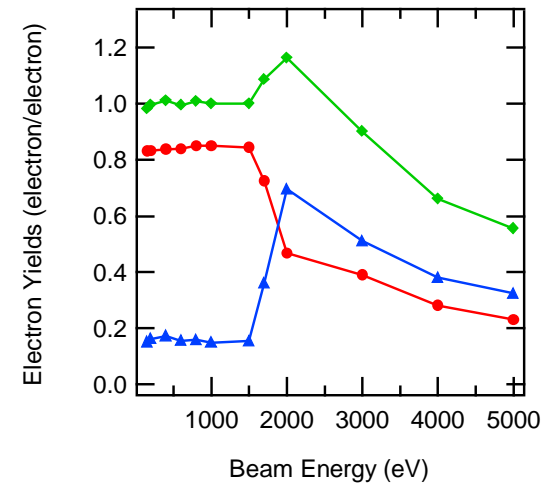

(c)

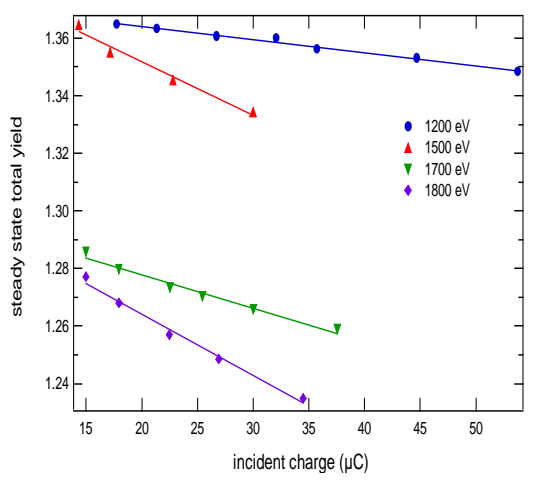

(f)

Fig 2. Measurements for $1.3 \mu \mathrm{m}$ anodized layer on an $\mathrm{Al} 2219$ alloy sample. (a) Reliable absolute total yields (5 $\mu$ s pulses with amplitudes $50 \mathrm{nA}$, each data point was the average of 10 pulses) were obtained with flooding the sample between yield measurements. Yield curve is fitted with various analysis models (see [5] for details). (b) Three consecutive total yield curves taken without neutralization. Each data point consists of one pulse $\left(\sim 10^{6}\right.$ electrons/pulse). The first $(\bullet)$, second $(\boldsymbol{\Delta})$,

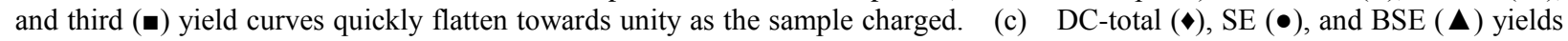
curves. Negative charging under DC bombardment continues up to $\mathrm{E}_{0} \approx 1600 \mathrm{eV}$ where the surface potential—estimated to be $35 \mathrm{eV}$ - exceeds the dielectric breakdown potential, with a field strength of $2 \cdot 10^{7} \mathrm{~V} / \mathrm{m}$. (d) Monitoring sample current confirmed dielectric breakdown at $1600 \mathrm{eV}$, where the sample began to conduct DC current. (e) SE DC spectra showed increasingly negative surface potentials at beam energies of $200 \mathrm{eV}(9 \pm 1 \mathrm{~V})$ (solid), $500 \mathrm{eV}(11 \pm 1 \mathrm{~V})$ (dash-dot), $1000 \mathrm{eV}(17 \pm 1$ V) (long dash), $1250 \mathrm{eV}(21 \pm 2 \mathrm{~V})$ (short dash), $1300 \mathrm{eV}(32 \pm 2 \mathrm{~V})$ (dot). Decline of SE peak magnitude at $1250 \mathrm{eV}$ indicated dielectric breakdown once more at a surface potential of $21 \pm 2 \mathrm{~V}$. (f) Dependence of evolving (quasi-)steady state total yields with incident energy and electron fluence. For $\mathrm{E}_{0}<\mathrm{E}_{2}$, the initial yield and slopes show no clear trends with energy (not shown; see [5]). For $E_{0}>E_{2}$, the initial yields values are seen to depend on energy, and the measured slopes were consistently negative.

(see Fig. 1(a)). The electric field from the negative charge again retards further incident electron penetration and acts to drive more low energy SE's from the sample, thereby enhancing the positive charge region [5,11]. The electric field from the positive charge region in turn acts to re-attract the lowest energy SE emitted from the surface (gray region in Fig. 1(d)), thereby establishing a shallow negative surface charge region. Because typical SE energy spectra (see Fig. 1(d)) peak at $\sim 3-5 \mathrm{eV}$, only a small net positive potential is required at the surface to attract sufficient electrons and thus reach current equilibrium [12].

These arguments explain why the SE and total yields (see, e.g., Fig. 2(a)) increase with increasing incident electron energy since higher energy incident electrons produce more SE's, but then fall off as the beam penetration depth exceeds the mean SE escape depth $(<10 \mathrm{~nm})$. The general arguments put forward here are in agreement with more sophisticated modeling [13-15] and recent measurements [16] of internal charge distributions resulting from electron bombardment of thin film insulators, as reviewed in [5].

Measuring shifts in the SE spectral emission peak provides a method for determining the sample surface potential of any material (particularly of charged insulator materials) while under continuous electron bombardment [5]. Sample potentials can have considerable affects on SE escape energies. Typically, a material with $0 \mathrm{~V}$ surface potential displays a $\mathrm{SE}$ emission peak near $2 \mathrm{eV}$ (see Fig. 1(h)). However, if the sample potential is negative, SE's will be accelerated away from the sample surface. The increased energy of escaping SE's can be observed in the spectra as a shift of the SE emission peak to higher energies. Alternatively, a few-volt positive sample potential will pull the majority of SE's back to the 
surface, shifting the SE spectra to lower energies [12].

\section{EXPERIMENTAL RESULTS}

Figures 1(b) and 1(f) show the evolution of the total yield as a function of incident charge-and internal charge accumulation-for incident energies $E_{1}<E_{0}<E_{2}$ and $E_{0}>E_{2}$, respectively. Between the crossover energies, the total yield asymptotically approaches unity exhibiting a decrease in total yield of $>2.5$ after only $2-5 \mu \mathrm{C} / \mathrm{m}^{2}$ of incident charge. Figures $1(\mathrm{~d})$ and $1(\mathrm{e})$ show the total yield decay constant as a function of beam energy, representing the rate at which the sample exponentially approaches a (quasi-)steady state with successive pulses. The decay constant decreased linearly with increasing $\mathrm{E}_{0}$ up to $\mathrm{E}_{0}=\mathrm{E}_{2}$. The decay for $E_{1}<E_{0}<E_{2}$ occurred for small incident charges, since positive surface potentials quickly re-attracted a significant portion of the SE spectrum (see Fig. 1(e)), an effect that was largely neutralized with low energy electron flooding. Beyond $E_{2}$, the decay constant remained small, but slightly positive as total yields and sample charge slowly approach their steady state values. This decrease of the charging rates for yields beyond $E_{2}$ resulted from two causes. First, the growing negative charge distribution diminished the landing energy of the incident electrons [2]; the increased total yields at lower landing energies further reduced negative charge accumulation and lowered the rate. Second, as internal charge builds up with each incident pulse, growing internal electric fields assist in SE transport towards the material surface. This resulted in consistent slow increases in the BSE yield, as SE in the high energy tail of the SE's emission spectra (gray region in Fig. 1(h)) were excited to emission energies $>50 \mathrm{eV}$ and converted to BSE. This effect was also observed in Fig. 2(c) as a sudden jump in BSE yield upon crossing $E_{2}$, which is attributed to switching sample potential polarity from positive to negative charging that accelerates escaping electrons to $>50 \mathrm{eV}$. Finally, as can be seen from Fig. 1(f), in this energy regime, flooding had little or no effect on the measured data since negative surface potentials repelled flooding electrons away from the sample surface.

Pulsed yield methods with alternating charge neutralization were used to make reliable and reproducible measurements of the absolute total yield curves of insulators [5,7]. These measurements showed very little evidence of the results of surface charge accumulation. Figure 2(a) shows a curve for an anodized layer using $5 \mu \mathrm{s}, 5 \mathrm{nA}$ pulses with electron flood charge neutralization between pulses.

However, even small charge accumulation was found to have substantial effect on yield curves. Three consecutive pulsed-total yield curves (5 $\mu \mathrm{s}$, 40-60 nA impulses) were taken without use of any neutralization techniques (see Fig. 2(b)). After just a few incident pulses, the subsequent yield curves were significantly dampened towards unity for $\mathrm{E}_{1}<\mathrm{E}_{\mathrm{o}}<\mathrm{E}_{2}$, even though the incident source was only depositing $\sim 10^{6}$ electrons/pulse over a beam-spot area of $\sim 10^{-6} \mathrm{~m}^{2}$. Treating the sample as a standard parallel plate capacitor (with an area of the beam spot), this amount of charge was estimated to change the surface potential by only 10-100 mV/pulse (positive). However, a significant portion of SE's are emitted with energies less than $5 \mathrm{eV}$ (see Fig. 1(d)) such that a cumulative positive surface potential of just $1 \mathrm{~V}$ can significantly suppress escaping SE's.

In the extreme, very large negative charge build up leads to dielectric breakdown. DC-yields were taken first using a continuous electron source at $\sim 20 \mathrm{nA}$ beam current. For energies ranging from $100 \mathrm{eV}$ to $1500 \mathrm{eV}$, the insulator quickly charged to a steady-state current equilibrium where the total yield reached unity, and no net current flowed to or from the sample (see Fig. 2(c)). However, above $\sim 1600 \mathrm{eV}$, a transition in the yield values occurred. As shown in Fig. 2(d), the sample current suddenly increased, indicating dielectric breakdown of the anodized coating.

Due to the repulsion of emitted SE's from the negatively charged sample, the SE peak is right-shifted to values corresponding to the sample potential (see Fig. 2(e)). A "false" SE emission peak is observed at $1.8 \pm 0.5 \mathrm{eV}$ in all the spectra caused by electron scattering from a grounded inner detector grid of our hemispherical grid retarding field analyzer, positioned between the sample and the retarding grid $[5,6]$. This false SE peak did not vary with sample type or bias, and was taken as a ground-reference potential for shifted sample SE peaks. Also, the height of the grid SE peak, with respect to the shifted sample SE peaks, provided a relative measure of the number of SE's emitted from the material. As shown in Fig. 2(e), as the beam energy was increased to $1250 \mathrm{eV}$ and $1300 \mathrm{eV}$ the surface potential reached $-21 \pm 2 \mathrm{~V}$ and $-31 \pm 2 \mathrm{~V}$, respectively and the SE peaks for these energies showed a significant decrease in relative amplitudes, indicative of the electrical breakdown. From the SE spectral data, the breakdown surface potential occurred near $-21 \mathrm{~V}$ (at beam energy $1250 \mathrm{eV}$ ), corresponding to a field strength of $\sim 2 \cdot 10^{7} \mathrm{~V} / \mathrm{m}$. This value was slightly lower than the calculated estimates from Fig. 2(d) and smaller than previously measured values [10]. The value may be slightly lower since the sample had previously been irradiated and broken down on the day before at $\sim 1600$ $\mathrm{eV}$ [5].

Once breakdown had occurred, the sample was irradiated for $10^{3} \mathrm{~s}$ at $5 \mathrm{keV}$ beam energy to determine if subsequent SE spectra would be affected by increased charge stored deep within the material. Subsequently, the incident beam was once again lowered and SE spectra were measured. The sample potentials measured at both at $500 \mathrm{eV}$ and $1000 \mathrm{eV}$ no longer showed dependence on incident beam energy (contrast Fig. 2(e)), but remained locked at $-8 \pm 1 \mathrm{~V}$. This demonstrated hysteresis in the sample emission, where 
residual charge from the high-energy incident beam remained trapped, keeping the sample potential at a negative value regardless of subsequent lower-energy electron irradiation between the crossover energies where positive surface charging should have occurred.

When all pulsed yields had been measured, the electron gun was turned to continuous emission to deposit large amounts of charge in the material, and the evolution of the steady state total yields were monitored as a function of total incident electron fluence and energy. The incident current varied between 20-30 nA (depending on the energy). The sample was irradiated up to $2 \cdot 10^{3} \mathrm{~s}$ for each energy, and total yields were plotted versus cumulative incident electron charge ( $\mathrm{I}_{0} \cdot$ time). For $\mathrm{E}_{0}<\mathrm{E}_{2}$, their existed no clear dependence between the total yields and incident charge (not shown here; see [5]). However, for $E_{0}>E_{2}$, total yields consistently decreased (slowly) with incident electron charge, as shown in Fig. 2(f). The rate of decrease was only $2-3 \%$ over $30-50 \mu \mathrm{C}$ of incident charge, but the trend was very consistent for each steady state yield set taken beyond $E_{2}$. It did not appear from the data that the slope magnitudes depended on incident energy, but the initial magnitudes of the steady state yields did. These data were consistent with predictions from Cazaux [13] and Reimer [2] that steady state yields should decrease with incident electron irradiation due to radiation induced conductivity as well as the additional defects and electron trapping that are created by the incident beam and high internal electric fields due to internal charge build up.

\section{ACKNOWLEDGMENTS}

The work presented was supported by the NASA Space Environments and Effects (SEE) Program, a NASA Graduate Student Research Fellowship, and Rocky Mountain NASA Space Grant Consortium Fellowships. Samples were supplied by NASA Marshall Space Flight Center [10] and Sheldahl Technical Materials.

\section{REFERENCES}

[1] D. Hastings, H. Garrett, Spacecraft-Environment Interactions, New York, NY: Cambridge Press, 1996.

[2] L. Reimer, Scanning Electron Microscopy. Physics of Image Formation and Microanalysis, New York, USA: Springer-Verlag, pp. 119-121, 1985.

[3] H. Seiler, "Secondary electron emission in the scanning electron microscope,” J. Appl. Phys., vol. 54 no. 11, pp. R1-R18, 1983.

[4] C.D. Thompson, V. Zavyalov, J.R. Dennison, J. Corbridge, "Electron Emission Properties of Insulator Materials Pertinent to the International Space Station," Proc. of the $8^{\text {th }}$ Spacecraft Charging Tech. Conf., (Huntsville, AL, USA), 2004.

[5] C.D. Thompson, "Measurements of Secondary Electron Emission Properties of Insulators," Ph.D. dissertation, Utah State University, Logan, UT, 2004.

[6] C.D. Thomson, V. Zavyalov, J.R. Dennison,
"Instrumentation for studies of electron emission and charging from insulators," Proc. of the $8^{\text {th }}$ Spacecraft Charging Tech. Conf., (Huntsville, AL, USA), 2004.

[7] J.R. Dennison, C.D. Thomson, J. Kite, V. Zavyalov, J. Corbridge, "Materials Characterization at USU: Facilities and Knowledgebase of Electronic Properties Applicable to Spacecraft Materials," Proc. $8^{\text {th }}$ Spacecraft Charging Tech. Conf., (Huntsville, AL, USA), 2004.

[8] I. Krainsky, W. Lundin, W.L. Gordon, R.W. Hoffman, Secondary Electron Emission Yield Annual Report for Period July 1, 1980 to June 30, 1981, Case Western Reserve University, (Cleveland, OH, USA), 1981, unpublished.

[9] JR Dennison, W.-Y. Chang, N. Nickles, J.T. Kite, C.D. Thomson, J. Corbridge, C. Ellsworth, "Final Report Part II1: Materials Reports," NASA Space Environ. and Effects Program, "Electronic Properties of Materials with Application to Spacecraft Charging," September 2002. Published by NASA electronically at http://see.msfc.nasa.gov/scck/.

[10] T. Schneider, NASA Marshall Space Flight Center, Materials, Processes, and Manufacturing Department, (private communications), 2003.

[11] X. Meyza, D. Goeuriot, C. Guerret-Piecout, D. Treheux, H.-J. Fitting, "Secondary Electron Emission and Self-consistent Charge Transport and Storage in Bulk Insulators: Application to Alumina,” J. Appl. Phys., vol. 94, pp. 5384-5392, 2003.

[12] N. Nickles, R.E. Davies, J.R. Dennison, "Applications of Secondary Electron Energy- and Angular-Distributions to Spacecraft Charging," Proc. of the $8^{\text {th }}$ Spacecraft Charging Tech. Conf., (AFRL Sc. Center, Hanscom AFB, MA, USA), 2000.

[13] J. Cazaux, "Some considerations on the secondary electron emission $\delta$, form $\mathrm{e}^{-}$irradiated insulators," $J$. Appl. Phys., vol. 85, no. 2, pp. 1137-1147, 1999.

[14] A. Melchinger, S. Hofmann, "Dynamic double layer model: Description of time dependent charging phenomena in insulators under electron beam irradiation,” J. Appl. Phys. vol. 78, pp. 6224-32, 2003.

[15] A.R. Frederickson, "Radiation Induced Electrical Current and Voltage in Dielectric Structures,” Phys. Sc. Res. Papers No. 613, AFCRL-TR-74-0582, Air Force Cambridge Res. Labs, (Hanscom AFB, MA), 1974.

[16] H. Saito, T. Urabe, Y. Tanaka, Y. Murooka, N. Tomita, “ Monitoring System for High Energy Cosmic Ray Observation in Space Environment using Advanced Electro-optic Pockels Techniques,” 2000 Conf. on Electrical Insulation and Dielectric Phenomena, IEEE, 694-697, 2000. 